\title{
Ecologia de comunidade de Bethylidae (Hymenoptera, Chrysidoidea) da Reserva Ecológica do Roncador, Brasília, Distrito Federal, Brasil ${ }^{1}$
}

\author{
Celso Oliveira Azevedo ${ }^{2}$ \\ José Luiz Helmer ${ }^{2}$
}

\begin{abstract}
Community ecology of Bethylidae (Hymenoptera, Chrysidoidea) from Reserva Ecológica do Roncador, Brasília, Distrito Federal, Brazil. Bethylidae specimens from the Reserve were studied in its ecological and faunistic aspects. The material was collected by Malaise and Window traps simultaneously in ten different areas of the Reserve during four years. The total number of genera and specimens were analyzed. Indices of diversity and evenness were used for characterizing the community ecology. Clustering analysis of localities and genera were provided. Nine genera of Bethylidae were found in the Reserve, being Pseudisobrachium Kieffer, 1904 and Apenesia Westwood, 1874 the most common ones. Window trap was more efficient than Malaise trap in terms of genus diversity.

KEY WORDS. Hymenoptera Bethylidae, community ecology, diversity, cerrado
\end{abstract}

Bethylidae são ectoparasitóides de Lepidoptera e Coleoptera de vida livre que vivem em situações crípticas, como casca ou troncos de árvores (brocadores), solo ou grãos (ASHMEAD 1893; EVANS 1964). Exibem adaptações para o mesmo habitat de seus hospedeiros, como corpo deprimido, asas reduzidas, pernas escavadoras (EvANS 1964). Cada hospedeiro pode receber uma ou várias ferroadas, o que induz a rápida paralisia permanente ou temporária (Evans 1964). Em algumas espécies de Bethylidae, a fềmea arrasta hospedeiro para locais isolados, onde alimenta-se de sua hemolinfa ou deposita ovos sobre eles (WiLliams 1919). Algumas fêmeas de Bethylidae permanecem juntas ao hospedeiro até que sua prole se desenvolva, e algumas vezes, mais de uma geração dessas vespas pode ser criada sobre um hospedeiro grande. Quando a fêmea é virgem, deposita ovos haplóides sobre o hospedeiro, onde se desenvolvem somente machos. A fêmea espera até que estes machos eclodam e a fertilizem para que possa, então, depositar sobre o mesmo hospedeiro uma segunda geração de ovos, desta vez haplóides e diplóides (EvANS 1964). Além do comportamento multiparasitário, alguns Bethylidae, como Sclerodermus domesticus Latreille, 1809, desenvolveram comportamento parasocial (CASALE 1991).

1) Trabalho com apoio financeiro parcial da Pró-reitoria de Pesquisa e Pós-graduação da Universidade Federal do Espírito Santo.

2) Universidade Federal do Espírito Santo, Departamento de Biologia. Avenida Marechal Campos 1468, 29040-090 Vitória, Espirito Santo, Brasil.

E-mail: cazevedo@npd.ufes.bre helmer@npd.ufes.br 
Estudos de estrutura de comunidade de insetos têm sido realizados por MARINONI \& DUTRA $(1991,1996,1997)$ e MARINONI et al. (1997) para o estado do Paraná. CoutinHo (1990) atenta para a falta de estudos da fauna de invertebrados em cerrados, que se resume, praticamente, a cupins e formigas, e enfatiza a existência de endemismo e de grande diversidade de insetos. O domínio do Cerrado apresenta peculiaridades climáticas, edáficas e hídricas, gerando condições específicas para o desenvolvimento de uma fauna e flora diversificada e muitas vezes endêmicas (Dias 1992).

Este trabalho tem como objetivo analisar a estrutura de comunidade e similaridade faunística entre diferentes áreas da Reserva Ecológica Roncador, Brasília, Distrito Federal, situada no domínio do Cerrado Brasileiro, tendo como objeto de estudo gêneros de Bethylidae, e ao mesmo tempo analisar a eficiência das armadilhas Janela e Malaise na captura de Bethylidae.

\section{MATERIAL E MÉTODOS}

O estudo foi realizado com material proveniente da Reserva Ecológica do Roncador (Brasília), área sob administração do Instituto Brasileiro de Geografia e Estatística (IBGE), onde ocorrem campo limpo, campo sujo, campo cerrado, cerradão, mata galeria e vereda. O material coletado pertence à Coleção Entomológica do IBGE, unidade de Brasília e é proveniente de um projeto maior intitulado "Sinecologia dos Insetos do Cerrado, sensu lato", realizado sob coordenação do Dr. Bráulio Dias.

Nesse projeto, foram amostradas dez áreas da Reserva, a saber: campo limpo sul, campo limpo norte, campo sujo sul, campo sujo norte, campo cerrado sul, campo cerrado norte, cerradão (mais precisamente, cerrado denso), vereda, ecótone mata/ cerrado e mata ciliar (Fig. 1), com uma armadilha Janela e uma armadilha Malaise em cada área durante quatro anos contínuos, de 1979 a 1983.

A armadilha Janela utilizada consistiu de uma placa de acrílico $72 \times 72 \mathrm{~cm}$ com uma moldura de alumínio perfilado de $80 \times 80 \mathrm{~cm}$, apoiada por um cocho de alumínio de $80 \mathrm{~cm}$ de comprimento por $10 \mathrm{~cm}$ de profundidade e $21 \mathrm{~cm}$ de largura da base e $34 \mathrm{~cm}$ no topo. Este cocho com um orifício (respiro para água da chuva) em cada lateral a $8 \mathrm{~cm}$ de altura. A armadilha foi instalada ao nível do solo e fixada por dois cordões laterais. A cada visita, era trocada a solução de água, detergente e conservante do cocho, e a placa de acrílico era pulverizada com inseticida.

A armadilha Malaise utilizada consistiu de uma tenda de malha fina, branca no teto e preta nas demais partes, com 2,05 $\mathrm{m}$ de comprimento, 1,50 $\mathrm{m}$ de altura na parte frontal e $1,10 \mathrm{~m}$ na parte posterior, a face frontal com $1,00 \mathrm{~m}$ de largura e a posterior com $1,10 \mathrm{~m}$, o teto com $0,90 \mathrm{~m}$ de largura e 2,00 $\mathrm{m}$ de comprimento; ápice da face frontal com um tubo com um orifício, no qual estava acoplado o suporte do frasco coletor. A armadilha foi instalada ao nível do solo e fixada por um cordão frontal, um posterior e quatro laterais. A cada visita, era trocada a solução de água e conservante do frasco coletor.

Ambas armadilhas eram visitadas quinzenalmente com recolhimento do material coletado. 


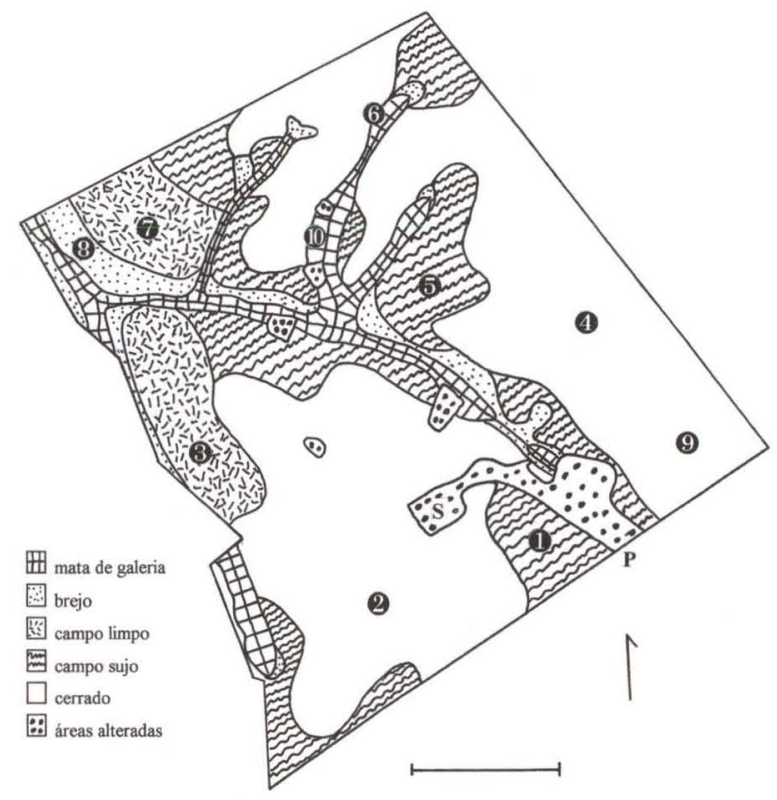

Fig. 1. Mapa da Reserva Ecológica do Roncador, Brasilia, Distrito Federal. (N) Norte, (P) portão de entrada, (S) sede, (1-10) sitios amostrais: (1) campo sujo sul, (2) campo cerrado sul, (3) campo limpo sul, (4) campo cerrado norte, (5) campo sujo norte, (6) ecótone cerrado/mata, (7) campo limpo norte, (8) vereda, (9) cerradão, (10) mata. Escala $=1 \mathrm{~km}$.

Em laboratório, o material capturado foi triado e todos os Bethylidae foram separados e montados para este estudo.

Todos os exemplares foram identificados em nível de gênero. Foi elaborada uma tabela em que foram listados os gêneros, com o número total de exemplares capturados em cada um dos dez sítios amostrais para cada tipo de armadilha utilizada (Tab. I). Com base nesses dados, foram feitos os estudos de estrutura de comunidade e similaridade faunística entre as localidades e análises estatísticas.

Com base nos dados de abundância de gêneros de Bethylidae, capturados pelas armadilhas Janela e Malaise, foi feita a caracterização da estrutura da comunidade através das medidas de diversidade de táxons utilizando-se o índice de Margalef $\left[D_{a}=(S-1) / \log (N)\right]$, índice de Simpson $\left[D_{\mathrm{S}}=1-\sum n_{i}\left(n_{i}-1\right) / N(N-1)\right]$, índice de diversidade de Brillouin $\left[H=\left(\log N !-\sum \log n_{i} !\right) / N\right]$, índice de Shannon $\left(H^{\prime}=\right.$ $-\sum p_{i} \log p_{i}$ ), e medidas de uniformidade usando os índices de Shannon (J' = $\left.\mathrm{H}^{\prime} / \mathrm{H}_{\max }{ }^{\prime}\right)$ e Simpson $\left(\mathrm{E}_{\mathrm{s}}=\mathrm{D}_{\mathrm{S}} / \mathrm{D}_{\max }\right)$; a variância de $H^{\prime}$ pelo método de Hutcheson $\left[\mathrm{s}^{2}=\left(\Sigma \mathrm{f}_{\mathrm{i}} \ln \left(\mathrm{f}_{\mathrm{i}}\right)^{2}-\left(\sum \mathrm{f}_{\mathrm{i}} \ln \left(\mathrm{f}_{\mathrm{i}}\right)\right)^{2} / \mathrm{n}\right) / \mathrm{n}^{2}\right]$ (BROWER \& ZAR 1984); índice de dominância de Berger-Parker $\left(\mathrm{BP}=\mathrm{N}_{\max } / \mathrm{N}\right)$ (SouTHwoOD 1978) e uniformidade de Berger \& Parker (UBP $=1 / \mathrm{BP})$ (apud MARINONI \& DUTRA 1996).

As análises de agrupamento foram processadas no programa Fitopac (versão 2.0) (SHEPHERED 1995). Para agrupamento dos ambientes foi utilizada a matriz da tabela I que contém os dados de estrutura da comunidade. Inicialmente, foram logaritimizados os dados de número de indivíduos e posteriormente estandardizou- 
se todos os dados da estrutura de comunidade. Para análise de agrupamento dos gêneros foram utilizados dados da tabela I. As análises de agrupamento, de locais e gêneros, foram realizadas separadamente para as armadilhas Malaise e Janela, utilizando-se o coeficiente de correlação, correlação linear e UPGMA como método de agrupamento.

Tab. I. Número de indivíduos capturados de cada gênero de Bethylidae por armadilha Malaise e Janela em cada sítio amostral na Reserva. (Pse) Pseudisobrachium, (Ape) Apenesia, (Dis) Dissomphalus, (Epy) Epyris, (Rha) Rhabdepyris, (Ani) Anisepyris, (Hol) Holepyris, (Cep) Cephalonomia, (Gon) Goniozus.

\begin{tabular}{|c|c|c|c|c|c|c|c|c|c|c|c|}
\hline Ambiente & Armadilha & Pse. & Ape. & Dis. & Epy. & Rha. & Ani. & Hol. & Cep. & Gon. & Total \\
\hline \multirow[t]{3}{*}{ Campo sujo sul } & Malaise & 137 & 169 & 1 & - & - & 1 & - & - & - & 308 \\
\hline & Janela & 1198 & 539 & 26 & 26 & 42 & 81 & 3 & 4 & 31 & 1951 \\
\hline & Total & 1335 & 708 & 27 & 26 & 42 & 82 & 3 & 4 & 31 & 2258 \\
\hline \multirow[t]{3}{*}{ Campo cerrado sul } & Malaise & 265 & 114 & - & - & - & - & - & - & - & 379 \\
\hline & Janela & 120 & 59 & 6 & 25 & 34 & 13 & 3 & 3 & 5 & 268 \\
\hline & Total & 385 & 173 & 6 & 25 & 34 & 13 & 3 & 3 & 5 & 647 \\
\hline \multirow[t]{3}{*}{ Campo limpo sul } & Malaise & 16 & 11 & 1 & - & - & - & - & - & - & 28 \\
\hline & Janela & 25 & 1 & 2 & - & 2 & - & - & - & - & 30 \\
\hline & Total & 41 & 12 & 3 & - & 2 & - & - & - & - & 57 \\
\hline \multirow[t]{3}{*}{ Campo cerrado norte } & Malaise & 178 & 97 & - & - & 1 & - & - & - & - & 275 \\
\hline & Janela & 50 & 26 & 8 & 14 & 10 & 22 & 1 & 1 & 1 & 134 \\
\hline & Total & 228 & 123 & 8 & 14 & 11 & 22 & 1 & 1 & 1 & 410 \\
\hline \multirow[t]{3}{*}{ Campo sujo norte } & Malaise & 128 & 51 & - & 2 & - & 13 & - & - & - & 194 \\
\hline & Janela & 45 & 92 & 21 & 20 & 24 & 18 & 1 & - & 11 & 232 \\
\hline & Total & 173 & 143 & 21 & 22 & 24 & 31 & 1 & - & 11 & 426 \\
\hline \multirow[t]{3}{*}{ Cerrado/mata } & Malaise & 224 & 35 & - & - & 1 & 3 & - & - & - & 263 \\
\hline & Janela & 16 & 8 & 13 & 4 & - & 32 & 1 & - & - & 74 \\
\hline & Total & 240 & 43 & 13 & 4 & 1 & 33 & 1 & - & - & 337 \\
\hline \multirow[t]{3}{*}{ Campo limpo norte } & Malaise & 79 & 80 & - & 1 & 2 & - & - & - & - & 162 \\
\hline & Janela & 70 & 44 & 9 & 10 & 21 & 17 & 3 & - & 15 & 189 \\
\hline & Total & 149 & 124 & 9 & 11 & 23 & 17 & 3 & - & 15 & 351 \\
\hline \multirow[t]{3}{*}{ Vereda } & Malaise & 3 & 9 & - & - & 1 & - & - & - & - & 13 \\
\hline & Janela & 64 & 2 & 17 & - & 7 & 6 & 2 & - & 1 & 99 \\
\hline & Total & 67 & 11 & 17 & - & 8 & 6 & .2 & - & 1 & 112 \\
\hline \multirow[t]{3}{*}{ Cerradäo } & Malaise & 21 & 7 & - & - & - & - & - & - & - & 28 \\
\hline & Janela & 17 & 3 & 4 & 2 & - & 3 & - & - & - & 29 \\
\hline & Total & 38 & 10 & 4 & 2 & - & 3 & - & - & - & 57 \\
\hline \multirow[t]{3}{*}{ Mata } & Malaise & 3 & 3 & - & - & - & - & - & - & - & 6 \\
\hline & Janela & 1 & - & 8 & - & 1 & 2 & 1 & - & - & 13 \\
\hline & Total & 4 & 3 & 8 & - & 1 & 2 & 1 & - & - & 19 \\
\hline \multirow[t]{2}{*}{ Total } & Malaise & 1054 & 576 & 2 & 3 & 5 & 17 & - & - & - & 1657 \\
\hline & Janela & 1606 & 774 & 114 & 101 & 141 & 194 & 15 & 8 & 64 & 3017 \\
\hline Total & & 2660 & 1350 & 116 & 104 & 146 & 211 & 15 & 8 & 64 & 4674 \\
\hline
\end{tabular}

Foram calculados índices que caracterizam a estrutura da comunidade amostrada para cada ambiente e tipo de armadilha (Tab. II). Foi utilizado o teste de Wilcoxon (in SOKAL \& ROHLF 1979) para dados pareados para comparar as características das comunidade capturadas pelas armadilhas Malaise e Janela, uma vez que as armadilhas foram utilizadas do mesmo modo em todos os ambientes amostrados. 
Tab. II. Valores de diversidade, uniformidade e dominância da fauna de Bethylidae para cada sítio amostral da Reserva. (N) Números de indivíduos, (S) número de gêneros, $\left(\mathrm{H}^{\prime}\right)$ indice de diversidade de Shanon; $\left(J^{\prime}\right)$ indice de uniformidade de Shanon, $\left(\mathrm{D}_{a}\right)$ indice de Margalef, $(H)$ indice de diversidade de Brillouin, (Ds) índice de diversidade de Simpson, $\left(E_{s}\right)$ indice de uniformidade de Simpson, (BP) indice de dominância de Parker, (UPB) indice de uniformidade de Parker, $\left(s^{2}\right)$ variancia de $H^{\prime}$.

\begin{tabular}{|c|c|c|c|c|c|c|c|c|c|c|c|c|}
\hline Ambiente & Armadilha & $N$ & S & $\mathrm{H}^{\prime}$ & $J^{\prime}$ & $\mathrm{Da}_{\mathrm{a}}$ & $\mathrm{H}$ & $D_{s}$ & $E_{s}$ & $\mathrm{BP}$ & UBP & $\mathrm{s}^{2}$ \\
\hline \multirow[t]{2}{*}{ Campo sujo sul } & Malaise & 308 & 4 & 0,727 & 0,52 & 0,52 & 0,309 & 0,503 & 0,668 & 0,549 & 1,822 & 0,0775 \\
\hline & Janela & 1950 & 9 & 1,073 & 0,49 & 1,06 & 0,488 & 0,544 & 0,611 & 0,614 & 1,628 & 0,0447 \\
\hline \multirow[t]{2}{*}{ Campo cerrado sul } & Malaise & 379 & 2 & 0,612 & 0,88 & 0,17 & 0,262 & 0,422 & 0,841 & 0,699 & 1,430 & 0,1669 \\
\hline & Janela & 268 & 9 & 1,583 & 0,72 & 1,43 & 0,804 & 0,726 & 0,813 & 0,448 & 2,233 & 0,0485 \\
\hline \multirow[t]{2}{*}{ Campo limpo sul } & Malaise & 28 & 3 & 0,806 & 0,73 & 0,60 & 0,306 & 0,537 & 0,777 & 0,571 & 1,750 & 0,1230 \\
\hline & Janela & 30 & 4 & 0,626 & 0,45 & 0,88 & 0,231 & 0,306 & 0,394 & 0,833 & 1,200 & 0,1273 \\
\hline \multirow[t]{2}{*}{ Campo cerrado norte } & Malaise & 276 & 3 & 0,671 & 0,61 & 0,36 & 1,228 & 0,462 & 0,691 & 0,645 & 1,551 & 0,1087 \\
\hline & Janela & 133 & 9 & 1,695 & 0,77 & 1,64 & 0,741 & 0,778 & 0,869 & 0,376 & 2,660 & 0,0451 \\
\hline \multirow[t]{2}{*}{ Campo sujo norte } & Malaise & 194 & 4 & 0,854 & 0,62 & 0,57 & 0,357 & 0,494 & 0,655 & 0,660 & 1,516 & 0,1019 \\
\hline & Janela & 232 & 8 & 1,715 & 0,82 & 1,29 & 0,819 & 0,774 & 0,881 & 0,397 & 2,522 & 0,0519 \\
\hline \multirow[t]{2}{*}{ Cerrado/mata } & Malaise & 263 & 4 & 0,477 & 0,34 & 0,54 & 0,199 & 0,258 & 0,342 & 0,852 & 1,174 & 0,1072 \\
\hline & Janela & 74 & 6 & 1,456 & 0,81 & 1,16 & 0,581 & 0,730 & 0,865 & 0,432 & 2,313 & 0,0653 \\
\hline \multirow[t]{2}{*}{ Campo limpo norte } & Malaise & 162 & 4 & 0,784 & 0,57 & 0,59 & 0,329 & 0,521 & 0,691 & 0,494 & 2,025 & 0,0910 \\
\hline & Janela & 189 & 8 & 1,735 & 0,83 & 1,34 & 0,824 & 0,781 & 0,888 & 0,370 & 2,700 & 0,0541 \\
\hline \multirow[t]{2}{*}{ Vereda } & Malaise & 13 & 3 & 0,790 & 0,72 & 0,78 & 0,266 & 0,500 & 0,692 & 0,692 & 1,444 & 0,1317 \\
\hline & Janela & 99 & 7 & 1,146 & 0,59 & 1,31 & 0,494 & 0,549 & 0,634 & 0,646 & 1,547 & 0,0655 \\
\hline \multirow[t]{2}{*}{ Cerradão } & Malaise & 28 & 2 & 0,562 & 0,81 & 0,30 & 0,217 & 0,389 & 0.750 & 0,750 & 1,333 & 0,1846 \\
\hline & Janela & 29 & 5 & 1,240 & 0,77 & 1,19 & 0,454 & 0,633 & 0,764 & 0,586 & 1,706 & 0,0835 \\
\hline \multirow[t]{2}{*}{ Mata } & Malaise & 6 & 2 & 0,693 & 1,00 & 0,56 & 0,216 & 0,600 & 1,000 & 0,500 & 2,000 & 0,1345 \\
\hline & Janela & 13 & 5 & 1,179 & 0,73 & 1,56 & 0,376 & 0,628 & 0,725 & 0,615 & 1,625 & 0,0865 \\
\hline
\end{tabular}

\section{RESULTADOS E DISCUSSÃO}

Foi obtido um total de 4674 exemplares de Bethylidae (Tab. I), tendo sido identificados nove gêneros para a Reserva: Pseudisobrachium Kieffer, 1904, Apenesia Westwood, 1874, Dissomphalus Ashmead, 1893, Anisepyris Kieffer, 1905, Holepyris Kieffer, 1905, Epyris Westwood, 1832, Rhabdepyris Kieffer, 1904, Goniozus Förster, 1856 e Cephalonomia Westwood, 1833.

Pseudisobrachium e Apenesia foram os dois gêneros distintamente mais abundantes na amostragem com 2660 e 1350 espécimes capturados, o que representa $85,6 \%$ do total. Os outros sete gêneros juntos representam apenas $14,4 \%$ da amostragem total (Tab. I).

AzEvedo (1991) estudou a fauna de Bethylidae de cerrado de São Carlos (São Paulo) e encontrou resultados semelhantes, em que Pseudisobrachium e Apenesia também foram os mais coletados, porém, não tão distintamente como na presente amostragem. Neste estudo foram reconhecidos, também, nove gêneros, sendo que Lepidosternopsis Ogloblin, 1953 ocorreu apenas no cerrado de São Carlos, onde Cephalonomia esteve ausente.

A fauna de Bethylidae do cerrado de Rondonópolis (MT) apresenta Anisepyris como o gênero mais abundante (AZEVEDO 1996), que reconheceu os mesmos gêneros, exceto Cephalonomia.

\section{Sobre armadilhas $x$ Bethylidae}

A armadilha Janela capturou mais Bethylidae do que a armadilha Malaise, com 3017 dos 4674 exemplares de Bethylidae obtidos (Tab. I), o que representa $64,55 \%$ do total. Estes dados mostram que, na média, a armadilha Malaise capturou 1,14 Bethylidae/dia e a armadilha Janela 2,07 Bethylidae/dia. A armadilha Janela 
capturou muito mais Bethylidae do que a armadilha Malaise em sete ambientes, sendo em dois deles com pouca diferença (Tab. I).

Assim, apesar de ambas armadilhas interceptarem o vôo dos insetos, elas capturam os insetos de maneiras diferentes, uma vez que na armadilha Janela a placa de acrílico é pulverizada com inseticida e na armadilha Malaise os insetos têm de caminhar até o frasco coletor. Os resultados aqui obtidos e as análises dos agrupamentos supracitados ressaltam estas diferenças.

Poucos estudos têm sido publicados sobre a eficiência de armadilhas na captura de Bethylidae. NOYES (1989) e Perioto (comunicação pessoal) realizaram análises em nível de família para comunidades de himenópteros parasitóides em floresta tropical úmida e cerrado, respectivamente. NOYES (1989) obteve através da armadilha de intercepção de vôo 4,21 Bethylidae/dia, o que foi mais eficiente que a armadilha Janela no nosso experimento. Ambas armadilhas apresentam o mesmo princípio de captura, mas NOYES (1989) utilizou uma rede de 3,0 x 1,3 m e em nossa amostragem foi utilizada uma placa de acrílico de $0,8 \times 0,8 \mathrm{~m}$, assim NOYES (1989) obteve 1,08 Bethylidae/dia por metro quadrado de armadilha e em nossa amostragem 3,24. Perioto (comunicação pessoal) utilizou duas armadilhas de pano com 1 $\mathrm{m}^{2}$ de área útil e obteve 0,05 Bethylidae/dia por metro quadrado útil de armadilha.

Nestas mesmas amostragens, NOYES (1989) obteve 0,64 Bethylidae/dia em armadilha Malaise e Perioto (comunicação pessoal), usando armadilha suspensa, obteve 1,24 , enquanto em nossa amostragem obtivemos 1,14.

Deste modo, considerando estes métodos de coleta, ambas as áreas de cerrado apresentaram maior abundância de Bethylidae do que área de floresta tropical úmida e a armadilha Janela apresenta-se mais eficiente na captura de indivíduos.

\section{Sobre armadilhas $\mathrm{x}$ gêneros}

A armadilha Janela capturou mais espécimes de Pseudisobrachium e Apenesia do que a armadilha Malaise, embora esta última tenha capturado mais exemplares na maioria das áreas amostradas. Isto ocorreu pelo fato da armadilha Janela ter sido muito efetiva no campo sujo sul (Tab. I). Este fato também ocorreu para os demais gêneros.

Nenhum exemplar de Goniozus, Cephalonomia e Holepyris foi coletado através da armadilha Malaise, e para os gêneros Anisepyris, Rhabdepyris, Epyris e Dissomphalus a armadilha Malaise foi responsável pela coleta de apenas $8 \%, 3,4 \%$, 0,03 e $0,02 \%$ da amostragem, respectivamente.

Deste modo, a armadilha Janela capturou um número significativamente maior de gêneros $(\mathrm{p}<0,01)$ do que a armadilha Malaise em todos os ambientes, exceto no campo limpo sul (Tab. I). O número de gêneros capturados pela armadilha Janela variou de cinco a nove gêneros por ambiente amostrados e a Malaise de dois a quatro (Tab. I).

Os sítios amostrais campo sujo sul e do campo cerrado sul foram os pontos que apresentaram a maior variedade, com todos os nove gêneros, seguidos de campo cerrado norte campo sujo norte e campo limpo norte com oito gêneros. Mata, campo limpo sul e cerradão foram os pontos que apresentaram a menor variedade, com seis, cinco e cinco gêneros. 


\section{Sobre armadilhas $\mathrm{x}$ estrutura da comunidade}

O número de indivíduos capturados por ambiente pela armadilha Janela $(\mathrm{N}=3017)$ não foi significativamente diferente $(\mathrm{p}>0,05)$ do capturado pela armadilha Malaise $(\mathrm{N}=1657)$, pois houve três ambientes nos quais a captura de Malaise foi maior (Tab. III). Quando se fez a comparação utilizando-se o número de indivíduos totais de cada gênero a diferença foi significativa $(\mathrm{p}<0,01)$ pois em todos os gêneros a captura total de Janela foi maior do que Malaise. Para todos os gêneros, a armadilha Janela capturou mais exemplares do que a armadilha Malaise (Tab. I).

Tab. III. Comparação entre armadilhas Malaise e Janela pelo Teste de Wilcoxon para amostras pareadas.

\begin{tabular}{|c|c|c|c|}
\hline Característica da comunidade & $\mathrm{N}$ & Estatistico T & Significância \\
\hline Número de indivíduos (N) (ambientes) & 10 & 24 & NS \\
\hline Número de individuos $\mathrm{N}$ (gênero) & 9 & 0 & $\star \star$ \\
\hline Número de espécies (S) & 10 & 0 & ** \\
\hline Diversidade de Shannon ( $\left.\mathrm{H}^{\prime}\right)$ & 10 & 1 & $\star \star$ \\
\hline Equitabilidade de Shannon (J') & 10 & 27 & NS \\
\hline Indice de Margalef $\left(D_{a}\right)$ & 10 & 0 & $\star \star$ \\
\hline Diversidade de Brillouin $(H)$ & 10 & 11 & NS \\
\hline Diversidade de Simpson $\left(D_{s}\right)$ & 10 & 5 & * \\
\hline Equitabilidade de Simpson ( $E_{s}$ ) & 10 & 26 & NS \\
\hline Dominância de Berger \& Parker(BP) & 10 & 11 & NS \\
\hline Equitabilidade de BP (UBP) & 10 & 8 & NS \\
\hline Variância de $\mathrm{H}^{\prime}\left(\mathrm{s}^{2}\right)$ & 10 & 1 & $\star \star$ \\
\hline
\end{tabular}

Os valores dos índices de diversidade, Shannon, Margalef e Simpson, obtidos a partir das captura de Janela e Malaise, foram significativamente diferentes $(\mathrm{p}<0,01,0,01$ e 0,05 , respectivamente) (Tab. II e III), com Janela capturando uma amostra mais diversificada. Os valores de uniformidade ou uniformidade relativa a estes índices mostraram que eles não são significativamente diferentes entre si ( $p>0,05$ ), indicando que embora estas armadilhas capturam composição faunística diferente quanto ao número de indivíduos e gêneros, a uniformidade das armadilhas em relação aos gêneros capturados permanece semelhante.

O índice de diversidade de Brillouin, dominância de Berger \& Parker e seu equivalente índice de uniformidade apresentaram valores que não podem ser considerados significativamente diferentes $(\mathrm{p}>0,05)$ quando se compara armadilha Janela com Malaise (Tab. II e III).

Pelo dados obtidos, pode-se dizer que a armadilha Janela é capaz de capturar uma fauna mais representativa do ambiente amostrado do que a armadilha Malaise pois, além de capturar uma maior quantidade de gêneros distintos, os valores de diversidade foram significativamente maiores (Tab. II e III).

\section{Análise de agrupamento dos sítios amostrais}

O sítio amostral do campo sujo sul foi o local onde houve a maior captura (2258 exemplares) seguido do campo cerrado sul (647) e campo sujo norte (426). O local de menor captura foi a mata com apenas 19 exemplares (Tab. I).

A análise deste agrupamento com os dados numéricos obtidos a partir da armadilha Janela da tabela II formou dois blocos. Em um bloco reuniu-se as áreas 
de campo sujo norte, campo limpo norte, as duas de campo cerrado e ecótone, neste caso as duas primeiras áreas se ligaram em um nível de similaridade muito elevado e as áreas campo cerrado norte e sul em um nível um pouco inferior. Estas quatro áreas se ligaram ao ecótone em um nível de similaridade mais baixo. Assim, neste bloco as áreas de cobertura vegetal menos densa ficaram distintamente agrupadas (Fig. 3).

No outro bloco reuniu-se as áreas de cobertura vegetal mais densa, cerradão e mata, mais úmida, vereda, além das áreas de campo limpo sul e campo sujo sul. Neste caso, as áreas de mata e cerradão, que possuem cobertura vegetal mais densa, se separam das demais.

Assim, de maneira geral, houve uma pequena tendência de aproximação de ambientes com cobertura vegetal mais densa e/ou úmidos (no caso vereda) de um lado e de ambientes com cobertura vegetal menos densa.

A análise deste agrupamento, utilizando-se os dados da tabela II, com a estrutura da comunidade capturada por armadilha Malaise mostrou a mesma tendência de aproximação de áreas mais úmidas e densas (Fig. 2).

Os dados numéricos da tabela I e as análises de agrupamentos supracitadas indicam, de maneira geral, que a abundância de exemplares e riqueza de gêneros das áreas de campo sujo (com $48,31 \%$ do total coletado) e campo cerrado foram maiores e nas vegetações com aspecto físionômico mais denso como as áreas de mata e cerradão foram os menores. Campo sujo e campo cerrado são tipos de vegetação do domínio do cerrado menos complexos do que cerradão ou mata galeria. Assim, seria esperado que nesses últimos fossem coletados mais exemplares e as comunidades tivessem diversidade maior, em função de uma possível relação entre a complexidade estrutural dos diferentes estágios serais de cerrado com a diversidade e quantidade de Bethylidae. A não ocorrência desta relação pode ser explicada pelo fato da Reserva Ecológica do Roncador ser pequena e apresentar todos os estágios serais de cerrado com áreas reduzidas, o que facilita o deslocamento de insetos entre estas vegetações. Outro fator que pode ajudar a explicar os resultados obtidos é que ambas as armadilhas utilizadas são de intercepção de vôo, o que as tornam mais eficientes em vegetações um pouco mais abertas.

\section{Análise de agrupamento dos gêneros}

A análise deste agrupamento com os dados numéricos obtidos a partir da armadilha Malaise (Fig. 4) aproximou Pseudisobrachium e Apenesia em um nível de similaridade de 0,64 e estes a Dissomphalus em um nível distintamente mais baixo. Anisepyris e Epyris se aproximaram em um nível de similaridade de 0,84 e estes se ligaram aos três primeiros por um nível de similaridade negativo. Rhabdepyris se ligou ao restante do bloco por um nível de similaridade ainda mais baixo.

Já a análise deste agrupamento com os dados numéricos obtidos a partir da armadilha Janela (Fig. 5) aproximou Pseudisobrachium, Apenesia, Anisepyris e Goniozus em um bloco. Pseudisobrachium e Apenesia se ligaram em um nível de similaridade de 0,98 e estes a Anisepyris em 0,91, que por sua vez se ligaram a Goniozus em 0,71. Epyris, Rhabdepyris e Cephalonomia formaram um segundo bloco, em que os dois primeiros gêneros se ligaram em nível de similaridade de 0,93 . Estes blocos se ligaram sucessivamente a Holepyris e depois a Dissomphalus. 

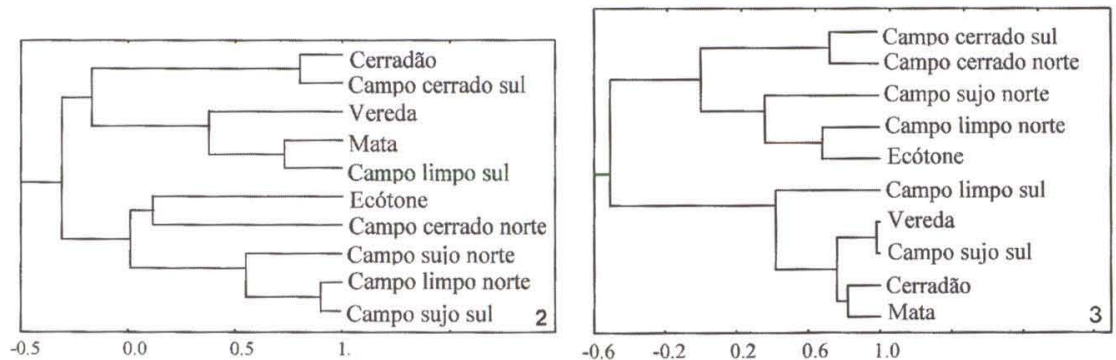

Distância da ligação

Figs 2-3. Análise de agrupamento dos sítios amostrais da Reserva usando-se dados de estrutura de comunidade da fauna de Bethylidae. (2) Armadilha Malaise; (3) armadilha Janela.

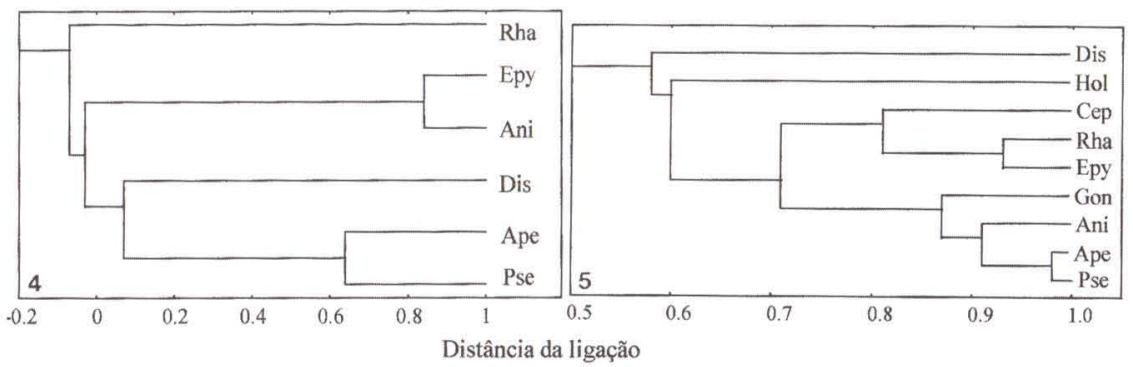

Figs 4-5. Análise de agrupamento dos gêneros capturados por armadilha presentes na Reserva. (4) Armadilha malaise; (5) armadilha Janela. (Pse) Pseudisobrachium, (Ape) Apenesia, (Dis) Dissomphalus, (Epy) Epyris, (Rha) Rhabdepyris, (Ani) Anisepyris, (Hol) Holepyris, (Cep) Cephalonomia, (Gon) Goniozus.

Os gêneros Pseudisobrachium, Apenesia e Dissomphalus (subfamília Pristocerinae) ficaram juntos na análise da armadilha Malaise. Estes são parasitóides de besouros mirmecófilos (EvANS 1961; KROMBEIN 1979) ou brocadores de madeira (KROMBEIN 1979), podendo atacar larvas de Cucujidae (Evans 1963) e Curculionidae (KIEFFER 1914). Há, também, muitas citações de ocorrência de suas espécies em ninhos de formigas de diversas subfamílias (ASHMEAD 1893; BRUCH 1916; EVANS 1961; 1964; WASMANN 1899). Os Bethylinae, aqui representados apenas por Goniozus e algumas espécies de Holepyris são parasitóides de larvas de Lepidoptera (EvANS 1977, 1978). Estes gêneros não ficaram agrupados, tendo se ligados em um nível de similaridade de 0,60, que foi baixo quando comparado com os dados da análise de agrupamento da armadilha Janela (Fig. 5). Os Epyrinae, incluindo algumas espécies de Holepyris, são parasitóides de larvas de Coleoptera de grãos armazenados, micófagos e de casca de árvores (EvANS 1964, 1969, 1977; WiLlians 1919). Estes gêneros ficaram juntos na análise de agrupamento da armadilha Malaise (Fig. 4) bem como na análise de agrupamento da armadilha 
Janela (Fig. 5), porém junto com Goniozus. É importante ressaltar que nada se conhece sobre os hospedeiros de Anisepyris e Rhabdepyris (Evans 1964), embora algumas espécies de Rhabdepyris já tenham sido coletadas em ninhos de formigas (KIEFFER 1914; EVANS 1964).

Assim, observou-se que o número de indivíduos capturados nos diferentes ambientes foi o fator que mais influenciou tais análises e não os aspectos relacionados à biologia dos gêneros.

\section{CONCLUSÕES}

Com base nesta amostragem realizada entre 1979 e 1983 com armadilhas Janela e Malaise na Reserva Ecológica do Roncador, chegou-se às conclusões abaixo.

A armadilha Janela é mais eficiente para a captura de Bethylidae tanto em número de indivíduos como de gêneros para a maioria das áreas amostradas.

Observa-se uma tendência dos ambientes com maior diversidade apresentarem uniformidade menor e vice-versa.

Nove gêneros ocorrem na Reserva, sendo Pseudisobrachium o mais abundante.

Não há relação entre aumento da complexidade estrutural da cobertura vegetal com a abundância de Bethylidae e diversidade genérica.

Há uma tendência, com base na fauna genérica de Bethylidae, de aproximação de ambientes com cobertura vegetal mais densa e/ou úmidos de um lado e de ambientes com cobertura vegetal menos densa.

Não há, de maneira clara, uma relação no agrupamento dos gêneros de Bethylidae e a biologia de cada um deles.

AGRADECIMENTOS. O primeiro autor agradece a hospitalidade de J. Dalmáceo e B. Dias durante suas duas estadas na Reserva Ecológica do Roncador; ao M.T. Teixeira e N.W. Perioto pelo auxílio na triagem do material aqui estudado.

\section{REFERÊNCIAS BIBLIOGRÁFICAS}

AsHMEAD, W.H. 1893. Monograph of the North American Proctotrypidae. Bul. U.S. Nat. Mus. 45: 472.

Azevedo, C.O. 1991. Comentário dos gêneros de Bethylidae (Hymenoptera, Aculeata) da região de São Carlos, SP, Brasil. An. Sem. Reg. Ecol. 6: 483-496. 1996. Sobre os Bethylidae (Hymenoptera, Chrysidoidea) da Reserva Indígena de Tadarimana, Rondonópolis, MT, Brasil. An. Sem. Reg. Ecol. 7: 111-119.

BRower, J.E. \& J.H. ZAR. 1984. Field and laboratory methods for general ecology. Dubuque, WCB, $2^{\text {nd }}$ ed., XII $+256 \mathrm{p}$.

BRUCH, C. 1916. Descripción de los himenópteros mirmecófilos pertenecientes á los Bethylidae. Physis 2: 19-23.

CASALE, A. 1991. Some notes on the parental and parasocial behavour of Sclerodermus domesticus Latreille (Hymenoptera, Bethylidae). Ethol. Ecol. \& Evol., special issue, 1: 35-38. 
CoutinHo, L.M. 1990. O Cerrado e a Ecologia do Fogo. Ciência Hoje 12 (68): 23-30.

DiAS, B.F.S. 1992. Cerrados: Uma Caracterização, p.11-25. In: B.F.S. DiAs (Coord.). Fundação Pró-Natureza. Alternativa de Desenvolvimento dos Cerrados: Manejo e Conservação dos Recursos Naturais Renováveis. Brasília, IBAMA, 97p.

Evans, H.E. 1961. A revision of the genus Pseudisobrachium in the North and Central America (Hymenoptera, Bethylidae). Bul. Mus. Comp. Zool. 126 (2): 211-318.

1963. A revision of the genus Apenesia in the Americas (Hymenoptera, Bethylidae). Bul. Mus. Comp. Zool. 130 (4): 249-359.

1964. A synopsis of the american Bethylidae (Hymenoptera, Aculeata). Bul. Mus. Comp. Zool. 132 (1): 1-222.

1966. A revision of the genus Anisepyris Kieffer (Hymenoptera, Bethylidae). Studia Entomol.9 (1-4): 1-120.

1969. A Revision of the Genus Epyris in the Americas (Hymenoptera, Bethylidae). Trans. Amer. Entomol. Soc. 95 (2): 181-352.

1977. A Revision of the Genus Holepyris in the Americas (Hymenoptera, Bethylidae). Trans. Amer. Entomol. Soc. 103: 531-579.

. 1978. The Bethylidae of America North of Mexico. Mem. Amer. Entomol. Inst. 27: 1-332.

KiefFer, J.J. 1914. Bethylinae. Das Tierreich 41: 228-595.

Krombein, K.V. 1979. Superfamily Bethyloidea, p.1203-1251. In: K.V. KroMBEIN; P.H. Hurd JR.; O.R. SMiTh \& B.O. BurKS (Eds). Catalog of Hymenoptera in America North of Mexico. Washington, Smithsonian Institution Press, Vol. 2., XVI+1199-2209.

MARINONI, R.C. \& R.R.C. DutRA. 1991. Levantamento da fauna entomológica do Estado do Paraná. I. Introdução. Situações climáticas e florística de oito pontos de coleta. Dados faunísticos de agosto de 1986 a julho de 1987. Revta bras. Zool. 8 (1-4): 31-73.

1996. Levantamento da fauna entomológica do Estado do Paraná. II. Ctenuchidae (Lepidoptera). Revta bras. Zool. 13 (2): 435-461.

1997. Famílias de Coleoptera capturadas com armadilha Malaise em oito localidades do estado do Paraná, Brasil. Diversidade alfa e beta. Revta bras. Zool. 14 (3): 751-770.

MARINONI, R.C.; R.R.C. Dutra \& M.M. CASAgRande. 1997. Levantamento da fauna entomológica do Estado do Paraná. III. Saturnidae (Lepidoptera). Revta bras. Zool. 14 (2): 473-495.

NOYES, J.S. 1989. A study of five methods of sampling Hymenoptera (Insecta) in a tropical rainforest, with special reference to the Parasitica. Jour. Nat. Hist. 23: 285-298.

SHEPHered, G.J. 1995. Fitopac, versão 2.0, manual do usuário. Campinas, Universidade Estadual de Campinas, 86p.

SOKAL, R.R. \& F.J. ROHLF. 1979. Biometria, principios y métodos estadísticos en la investigación biológica. Rosario, H. Blume, XII+832p.

Southwood, T.R.E. 1978. Ecological Methods. With Particular Reference to 
the Study of Insect Populations. London, Chapman \& Hall, XXIV+524p. WASMAnN, E. 1899. Die psychischen Fahigkeiten der Ameisen. Zoologica 11 (26): $1-133$.

WiLLIANS, F.X. 1919. Epyris extraneus Bridwell (Bethylidae), a fossorial wasp that preys on the larva of the tenebrionid beetle, Gonocephalum seriatum (Boisduval). Proc. Hawaiian Entomol. Soc. 4: 55-63.

Recebido em 06.VIII.1998; aceito em 07.X.1999. 